MATEC Web of Conferences 8, 01005 (2013)

DOI: $10.1051 /$ matecconf $/ 20130801005$

(C) Owned by the authors, published by EDP Sciences, 2013

\title{
Taming light with photonic lattices written by femtosecond laser
}

\author{
Matthieu Bellec $^{1, \dagger}$, Giorgos M. Nikolopoulos ${ }^{1}$ and Stelios Tzortzakis $^{1,2, *}$ \\ ${ }^{1}$ Institute of Electronic Structure and Laser, Foundation for Research and Technology Hellas (FORTH), Greece \\ ${ }^{2}$ Department of Materials Science and Technology, University of Crete, P.O. Box 2208, GR-71003 Heraklion, Greece \\ *stzortz@iesl.forth.gr
}

\begin{abstract}
Photonic lattices, arrays of evanescently coupled waveguides written by femtosecond laser, are often used to control the flow of light. Two cases are here considered experimentally. i) In the linear regime, a faithful transfer is achieved when the photonic structure is judiciously designed. ii) In the nonlinear regime, we show that photonic lattices support a new type of laser filaments, called lattice filaments, with controllable attributes.
\end{abstract}

Photonic lattices (PLs) are a remarkably simple and versatile tool to investigate various phenomena encountered in many areas of physics (e.g. Anderson localisation [1]). Besides their versatility, PLs allow to control the flow of ligth in both linear and nonlinear regime [2] and offer scalability and compatibility with the widespread silica technologies. Thus, they are expected to play a pivotal role in the route towards all-optical networking [3] and large scale quantum information processing [4].

Faithful communication Hamiltonian. - The faithful transfer of signals is a necessary requirement for further developments in these directions, and has thus attracted considerable interest. In the linear regime, one way to prevent the uncontrollable spread of the wavepacket is to judiciously engineer the coupling constants between adjacent waveguides (i.e. adjust the corresponding separation distance according to Fig. 1a). This scenario has been
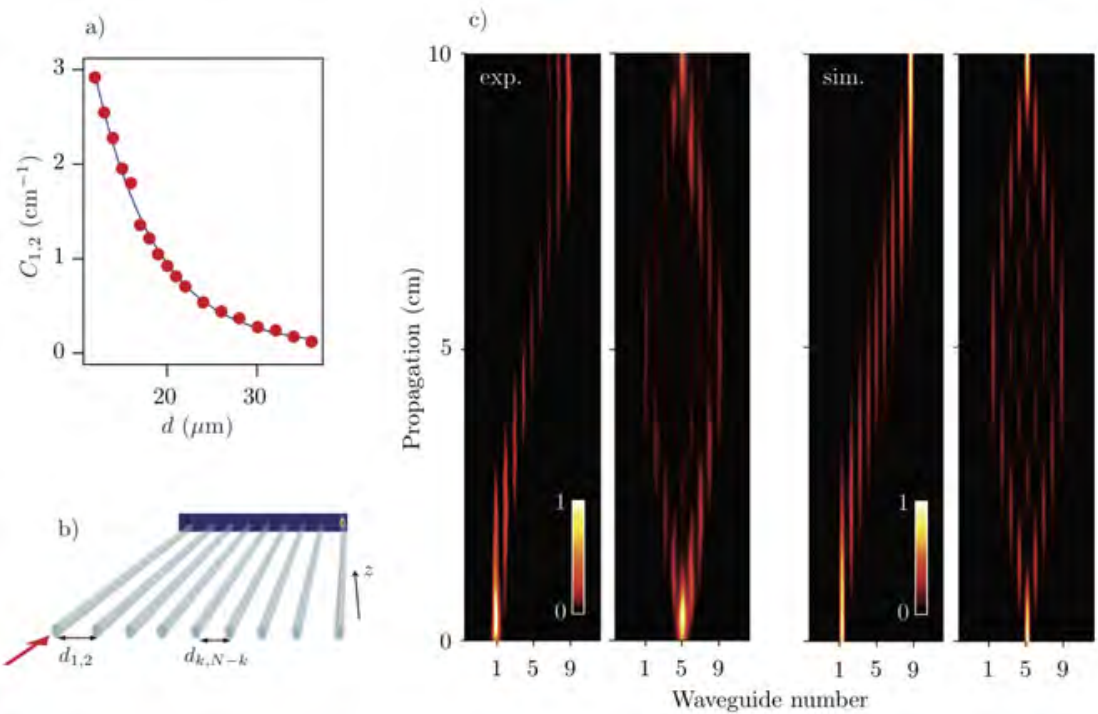

Fig. 1: a) Coupling strength $C$ versus waveguide separation $d$. b) Sketch of the photonic lattice with judiciously engineered couplings. c) Light propagation in an array of $N=9$ waveguides with coupling distribution given by eq. (1) when the first and fifth waveguide are initially excited. Left panels: Experiments. Right panels: Simulations. Adapted from [7]

also studied thoroughly in the context of quantum networks [5], and various faithful-communication (FC)

Hamiltonians have been proposed. In our case, in a photonic lattice of $N$ waveguides of fixed length $L$ (see Fig. 1b), faithful (ideally perfect) power transfer from the $k$ th to the $(N-k+1)$ th waveguide can be achieved if the couplings $C_{k, k \pm 1}$ between neighbouring waveguides $k$ and $k+1$ are chosen according to:

$$
C_{k, k+1}=C_{0} \sqrt{(N-k) k},
$$

†Present address: Laboratoire de Physique de la Matière Condensée, CNRS UMR 7336, Université Nice-Sophia Antipolis, 06108 Nice Cedex bellec@unice.fr 


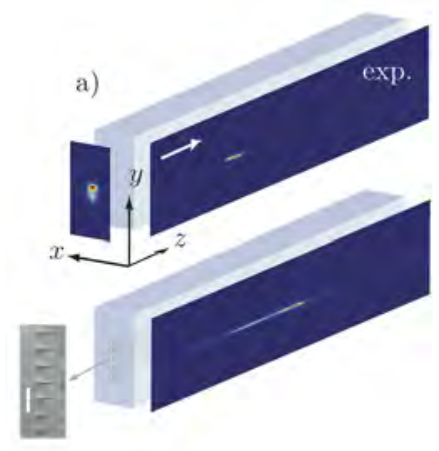

b)

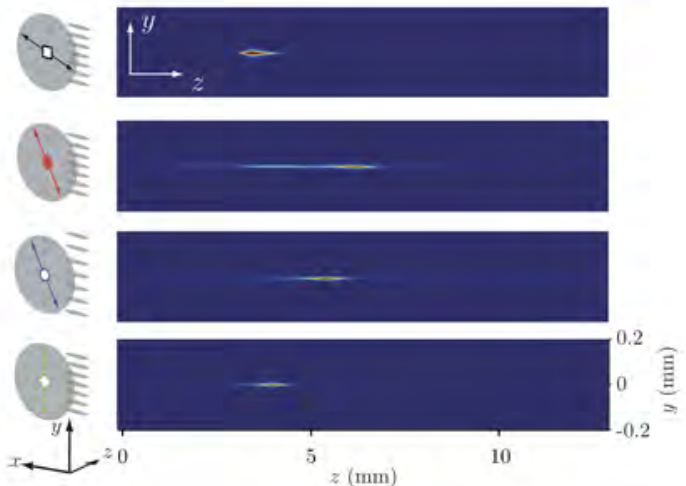

Fig. 2: Observation and tailoring of a lattice filament. a) top: A standard filament, bottom: a lattice filament. The $y z$ image size is $0.4 \times 13 \mathrm{~mm}$. b). Modification of the lattice period and input polarisation allows to tailoring the lattice filament attributes. Adapted from [10]

with $C_{0}=\pi /(2 L)$. Here, in the context of femtosecond-laser-written waveguide arrays [6], we present the first experimental realization of such a system. Our observations are compared to theoretical predictions (see Fig. 1c). The influence of disorder and losses are also discussed.

Lattice filaments. - Then, we considered a standard PL (i.e. with constant coupling) but in the nonlinear regime, more particularly where laser filamentation occurs. Filaments appear at laser pulse powers exceeding a critical power and result from a competition involving the optical Kerr effect, generation of free electrons, plasma induced defocusing, nonlinear losses and dispersion [8]. A control of this competition is extremely challenging since specific applications of filaments would request some of their features to be tuned along the propagation distance. However, high intensities within a standard filament make this task almost impossible with conventional optical elements. We demonstrate experimentally here that the techniques for stabilizing spatial solitons via the use of photonic lattices [2,9] extend advantageously to filamentation control [10]. Diffraction due to the lattice modifies the competition between linear and nonlinear effects that occur in a standard filament (SF). It prevents high intensities to be reached, thereby limiting nonlinear losses, by regularizing the energy flux from the periphery to the core of the filament. A nonlinear structure called intense lattice filament (LF) forms over a short propagation distance in the lattice (see Fig. 2a), with attributes that can be effectively tailored by adjusting the lattice properties (modulation depth and period), as depicted in Fig. 2b. We also shown that the LF attributes can be dynamically tailored through the interaction between filaments [10]. This opens the way for extensive all-optical control of the nonlinear propagation of intense ultrafast wave packets.

\section{References}

[1] T. Schwartz, G. Bartal, S. Fishman and M. Segev, "Transport and Anderson localization in disordered two-dimensional photonic lattices" Nature 446, pp. 52-55 (2007).

[2] D. N. Christodoulides, F. Lederer and Y. Silberberg, “Discretizing light behaviour in linear and nonlinear waveguide lattices” Nature 424, pp. 817-823 (2003).

[3] R. Keil, M. Heinrich, F. Dreisow, T. Pertsch, A. Tünnermann, S. Nolte, D. N. Christodoulides and A. Szameit, “All-optical routing and switching for three-dimensional photonic circuitry” Sci. Rep. 1, pp. 94 (2011).

[4] A. Politi, M. J. Cryan, J. G. Rarity, S. Yu, and J. L. O’Brien, “Silica-on-Silicon Waveguide Quantum Circuits” Science 320, pp. 646-649 (2008)

[5] A. Kay "Perfect, efficient, state transfer and its application as a constructive tool” Int. J. Quantum Inf. 08, 641 (2010).

[6] A. Szameit and S. Nolte, “Discrete optics in femtosecond-laser-written photonic structures” J. Phys. B 43, 163001 (2010).

[7] M. Bellec, G. M. Nikolopoulos and S. Tzortzakis, “A Faithful Communication Hamiltonian in Photonic Lattices” Opt. Lett. 37, pp. 4504-4506, (2012).

[8] A. Couairon and A. Mysyrowicz, “Femtosecond filamentation in transparent media” Phys. Rep. 441, pp. 47-189 (2007).

[9] N. K. Efremidis, J. Hudock, D. N. Christodoulides, J. W. Fleischer, O. Cohen and M. Segev, “Two-Dimensional Optical Lattice Solitons” Phys. Rev. Lett. 91, 213906 (2003).

[10] M. Bellec, P. Panagiotopoulos, D. Papazoglou, N. Efremidis, A. Couairon and S. Tzortzakis, “Observation and Optical Tailoring of Photonic Lattice Filaments" Phys. Rev. Lett. 109, 113905, (2012). 